\title{
La producción como origen del plusvalor en la teoría marxista
}

\author{
Production as Origen of Surplus-Value in \\ Marxist Theory
}

Produção como uma fonte de mais-valia na teoria marxista

Fahd Boundi Chraki *

DOI: https://doi.org/10.19053/01203053.v36.n64.2017.5598

Fecha de recepción: 18 de noviembre de 2016

Fecha de aceptación: 25 de mayo de 2017

\footnotetext{
* Investigador independiente. Licenciado en Ciencias Económicas, máster en Economía Internacional y doctor en Economía por la Universidad Complutense de Madrid. Madrid, España. Correo electrónico: fboundi@ucm.es inttp://orcid.org/0000-0002-4137-6202
} 


\section{Resumen}

El objetivo de la presente investigación es doble. Por una parte, consiste en dilucidar que la fuente misma de la ganancia, esto es, el plusvalor, dimana de la esfera de producción. Por otra, demostrar que la condición necesaria y suficiente para el sostenimiento del orden de sociedad capitalista reside en que entre el capital y el trabajo ha de mediar una relación de dominación expresada en la explotación de la fuerza de trabajo. Para este propósito, se confronta la teoría de Marx con el enfoque marginalista. Seguidamente, se descifra el secreto del plusvalor en la compraventa de fuerza trabajo, manifestando de esta suerte el carácter dual del sistema de cálculo marxiano. Finalmente, por medio del teorema marxiano fundamental se verifica la hipótesis de que la explotación de la fuerza de trabajo es condición necesaria y suficiente para que los capitalistas obtengan ganancias positivas.

Palabras clave: producción, capital y mano de obra, marxismo.

Clasificación JEL: B12, B13, B14, P10, P16. 
Apuntes CENES Volumen 36, Número 64

julio - diciembre 2017. Págs. 15-46

\section{Abstract}

The aim of this research is: 1) clarify the main source of profit, i.e., the surplus value, derives from the sphere of production; 2) show that the necessary and sufficient condition for sustaining capitalist society is that between capital and labor has to mediate a relationship based on the exploitation of the labor force. Firstly, Marx's theory will be confronted with the marginalist approach. Secondly, the secret of surplus-value is decrypted as the sale of labor force, showing in this way the dual character of Marxian system calculation. Finally, through the fundamental Marxian theorem it will be verified the hypothesis on the exploitation of the labor force as necessary and sufficient condition to obtain positive earnings.

Keywords: production, capital and labor, Marxism. 


\section{Resumo}

O objetivo desta pesquisa é: 1) esclarecer a principal fonte de lucro, ou seja, a mais-valia, deriva da esfera de produção; 2 ) mostra que a condição necessária e suficiente para sustentar a sociedade capitalista é que entre o capital eo trabalho tem que mediar uma relação baseada na exploração da força de trabalho. Em segundo lugar, o segredo da mais-valia é descriptografado como a venda da força de trabalho, mostrando assim o duplo caráter do cálculo do sistema marxista. Finalmente, através do teorema marxista fundamental, verifica-se a hipótese sobre a exploração da mão-de-obra como necessária e condição suficiente para obter ganhos positivos.

Palavras-chave: produção, capital e trabalho, Marxismo. 
Apuntes CENES Volumen 36, Número 64

julio - diciembre 2017. Págs. 15-46

\section{INTRODUCCIÓN}

Durante el lapso que media entre las fechas de enero de 1862 y julio de 1863, Marx se entregó a la ímproba tarea de escudriñar la teoría del plusvalor, tal y como había sido legada por la economía política clásica, para ulteriormente someterla a la más dura de sus críticas. De aquellos manuscritos nacería el que hoy es considerado como el libro IV de El Capital: las Teorías sobre la plusvalía ${ }^{1}$.

Baste señalar que la fuerza con la que inicia Marx (1956, p. 34) su crítica en el capítulo I de las Teorías sobre la plusvalía pone de manifiesto cuán confusos se encontraban los economistas que lo precedieron: "Antes de los fisiócratas, la plusvalía -es decir, la ganancia, bajo la forma de tal ganancia- se explicaba pura y simplemente a base del cambio por la mercancía en más de su valor".

No en vano, figuras tan representativas de la economía política moderna como Eugen von Bhöm-Bawerk (1876, 1891) y Keynes (1936) tuvieron a bien arrogarse esta errada concepción del origen del plusvalor para soslayar el

\footnotetext{
Aunque los manuscritos que constituyen las Teorías sobre la plusvalía fueron concluidos en 1863, la obra completa fue publicada por vez primera en 1905-1910 con la edición de Karl Kautsky. Sin embargo, la edición de Kautsky se caracteriza por la supresión de varios pasajes y la modificación deliberada de otros tantos, por lo que se ha preferido trabajar con la traducción de Fondo de Cultura Económica de 1980, la cual se encuentra basada en la edición alemana elaborada por Dietz en 1956-1959. Así pues, siempre que hagamos alusión a las Teorías sobre la plusvalía nos estaremos refiriendo para el libro I a la primera edición publicada en 1956, y para el libro II a su primera edición de 1959.
} 
primado de la producción capitalista de mercancías: la explotación de la fuerza de trabajo.

Mas, por otra parte, fueron los fisiócratas, observa Marx, los primeros en advertir que el punto de partida para desentrañar el origen del plusvalor emplazaba el riguroso análisis de la esfera de producción. Los fisiócratas, añade Marx, sentaron las bases objetivas sobre las que el capital se manifiesta dentro del proceso laboral $\mathrm{y}$, por tanto, pudieron determinar con alto grado de acierto las formas que adoptaba en la circulación. Así, de una parte, el capital asume la forma de capital fijo (avance primitive); de otra, toma la envoltura del capital circulante (avance annuelle).

Marx recalca, no obstante, que en los fisiócratas la fuente única del plusvalor es la renta de la tierra, en tanto que la ganancia del capitalista corresponde a una suerte de salario de grado superior que era pagado por el terrateniente. De tal forma, no debe asombrar que en el esquema de Quesnay (1758) la clase productiva sea la de los trabajadores agrícolas, en cuanto productores de plusvalor, mientras que los terratenientes son quienes se lo apropiaban al tratarse de la classe propriétaire.

Aun habiendo concentrado su análisis en la renta de la tierra, Marx no pudo por menos que elogiar la brillantez del sistema fisiócrata, en la medida en que este sistema rubricaba el origen del plusvalor dentro de la esfera de producción.

Esto es tanto más interesante cuanto que ayuda a comprender por qué en el transcurso de su investigación, Marx tuvo por bien tomar como punto de partida las reflexiones de los fisiócratas y, por extensión, las de Adam Smith (1776) y David Ricardo (1821), para con ello ulteriormente develar el secreto que se esconde detrás del origen de la ganancia del capital.

Así pues, a través de la teoría de Marx, el objetivo del presente capítulo es doble. i) Por una parte, consiste en dilucidar que la fuente misma de la ganancia, esto es, el plusvalor, dimana de la esfera de producción. ii) Por otra, demostrar que la condición necesaria $y$ suficiente para el sostenimiento del orden de sociedad capitalista es que entre el capital y el trabajo medie una relación de dominación, cuya expresión genuina sea la explotación de la fuerza de trabajo.

Tras este breve recorrido introductorio, debe señalarse que la investigación se estructura en cuatro apartados. En el primero, la explicación se centra en la crítica marxiana de la teoría de la utilidad marginal. En el segundo apartado se analiza la compraventa de la fuerza de trabajo como origen del plusvalor, descubriendo así el carácter 
dual del sistema de cálculo de Marx. En el tercero se profundiza en el teorema marxiano fundamental, según el cual la explotación de la fuerza de trabajo es la condición necesaria y suficiente para que los capitalistas obtengan ganancias positivas. En el cuarto y último apartado se extraen las conclusiones más relevantes.

CRÍTICA MARXIANA DE LA TEORÍA DE LA UTILIDAD MARGINAL

Cabe hacer notar que la lucidez de los fisiócratas es tanto más asombrosa cuanto más contrasta con el desconcierto que supone para el moderno sistema keynesiano localizar la fuente de la ganancia capitalista. Conviene citar en este respecto uno de los pasajes más polémicos de la Teoría general de Keynes (1936):

Es preferible considerar al trabajo, incluyendo, por supuesto, los servicios personales del empresario y sus colaboradores, como el único factor de la producción que opera dentro de un determinado ambiente de técnica, recursos naturales, equipo de producción y demanda efectiva. Esto explica, en parte, por qué hemos podido tomar la unidad de trabajo como la única unidad física que necesitamos en nuestro sistema económico, aparte de las de dinero y de tiempo. (1936, p. 213)
La controversia suscitada por esta cita deriva de la interpretación que hace de ella Axel Kicillof $(2005,2009)$, quien arguye que Keynes, al igual que Ricardo y que Marx, sostuvo en su Teoría general que la fuente única del valor $\mathrm{y}$, por extensión, del plusvalor, es el trabajo humano.

Sin embargo, Kicillof yerra al presuponer la existencia de un nexo que une la teoría del valor de Keynes con la teoría valor trabajo de Ricardo y Marx. En primer lugar, Keynes vincula la ganancia del capital a la escasez, esto es, cuanto más escasas son las mercancías tanto mayores serán sus precios respecto a sus valores. Ello significa que la ganancia de los capitalistas deriva de la diferencia entre el precio de mercado y el valor de las mercancías.

En segundo lugar, en Keynes subyace una teoría basada en el cálculo marginal psicológico, en la medida en que esta determina qué parte del producto global se acumula para posteriormente reinvertirse y cuál se dedica para satisfacer las necesidades de consumo.

Esto último entrelaza la Teoría general con los Principios de economía política de Alfred Marshall (1890), quien postuló que la ganancia de los capitalistas se encontraba sujeta a la productividad del capital. Más exactamente, en la teoría marginalista de Marshall la ganancia capitalista depende del rendimiento marginal del capital y de 
la tasa marginal para renunciar el uso presente de la renta.

$\mathrm{O}$, en otras palabras, a medida que se incrementa el capital, su productividad marginal irá decreciendo. En consecuencia, la retribución de los capitalistas, es decir, su ganancia, dependerá de la productividad marginal del capital.

Nótese cuán de similar es la teoría marginalista Marshall con la concepción de Nassau W. Senior (1836), quien hubo de anticipar el postulado ampliamente extendido por la escuela neoclásica sobre el origen de la ganancia derivada del sacrificio que supone para el capitalista el abstenerse de consumir.

Aunque Keynes subrayó con insistencia que la eficiencia marginal de capital no debía ser confundida con la productividad marginal del capital, el mecanismo expuesto en la Teoría general es, en esencia, muy similar al de Marshall. Empero, Keynes incluyó un factor ausente en la teoría neoclásica: la preferencia por la liquidez de los capitalistas.

El economista de Cambridge aduce que los capitalistas tienden a conservar una parte de su renta en forma líquida, ya sea en dinero o bien en forma de otro sustitutivo que cumpla con sus funciones. De esta suerte, los capitalistas se enfrentan, según Keynes, a la disyuntiva sobre qué hacer con su ahorro: i) invertirlo productivamente, o ii) conservarlo en forma líquida.

De acuerdo con Keynes, esta preferencia por la liquidez ha de afectar las ganancias futuras de los capitalistas, en la medida en que si los capitalistas resuelven conservar parte de sus ahorros en forma de dinero, la demanda de inversión y la demanda de consumo disminuirán. Luego, la demanda efectiva se contraerá hasta dar paso a una sobrecapacidad productiva.

En vista de esto, difícilmente podrá reconciliarse el corpus teórico de Keynes con la teoría valor trabajo de Ricardo y Marx, pues el sistema keynesiano no deja lugar a la duda: la ganancia del capital no puede originarse en la esfera de producción. Keynes sitúa, en definitiva, el origen de la ganancia en la esfera de circulación, tal y como lo hiciera a la sazón Malthus.

En este sentido, Keynes (1933, p. 23) escribe que: "Según la concepción de Malthus, llena de sentido común, precios y beneficios son determinados primariamente por algo que él describe, aunque nunca con demasiada claridad, como la demanda efectiva". Keynes (1933), por otra parte, nunca ocultó la animadversión que le generaba en su persona la figura de Ricardo:

¡Si Malthus y no Ricardo hubiera sido el tronco del que brotó la 
ciencia económica del siglo XIX, cuánto más sabio y rico sería hoy el mundo! Tenemos que redescubrir laboriosamente y hacer brotar a través de las oscuras capas de nuestra desorientada educación lo que nunca debió dejar de ser evidente. (p. 37)

En el sistema keynesiano no hay en forma alguna cabida para una teoría de la determinación de la ganancia capitalista basada en la teoría de la explotación de la fuerza de trabajo. Vale decir, asimismo, que para Keynes el primum movens del modo capitalista de producción es la satisfacción de las necesidades de consumo, mas no así la valorización del capital, como sostiene Marx en El Capital.

La proposición según la cual la producción capitalista sirve en última instancia para satisfacer las necesidades individuales de consumo constituye una fuerte conexión entre la teoría keynesiana y la teoría marginalista. Así, por ejemplo, Léon Walras (1874) desarrolló una teoría del valor basada en la escasez, en tanto y en cuanto, el valor se encuentra determinado por la escasez de los bienes respecto de las necesidades de consumo de los individuos.

Por su parte, Böhm-Bawerk (1876) arguyó que la producción capitalista de mercancías tiene como fin único satisfacer el consumo individual. De este modo, el economista austriaco consideró de escaso interés el estudio de la esfera de producción, debiéndose focalizar el análisis en la esfera de circulación, por cuanto el valor y el plusvalor (interés en sentido de BöhmBawerk) nacen de: "la dependencia de la satisfacción de las necesidades hu-manas por los bienes" (BöhmBawerk, 1876, p. 39).

Böhm-Bawerk agrega, asimismo, que el plusvalor -o interés, siguiendo su terminología-surge de la diferencia entre el valor presente y el valor futuro de los bienes. $\mathrm{O}$, más concretamente, los individuos muestran una mayor preferencia por los bienes presentes que por los bienes futuros.

La razón de ello, según el propio Böhm-Bawerk, se debe a: i) causas psicológicas derivadas de la incertidumbre con respecto al futuro; ii) causas técnicas determinadas por las condiciones de producción. Al igual que en Malthus, Keynes, Marshall y Walras, la concepción austriaca sitúa la génesis del plusvalor en la esfera de circulación.

Pero esto no es todo. Baste reseñar que en la economía política moderna ha sido un tema harto recurrente la posibilidad de que Marx tuviera conocimiento sobre la teoría de la utilidad marginal desarrollada por Menger, Jevons, Walras, Marshall o BöhmBawerk, habida cuenta de que la revolución marginalista se inició en 1870 . 
De haber profundizado Marx en dicha teoría, cabría esperar su consecuente respuesta que, desafortunadamente, no aparece expuesta explícitamente en parte alguna de los tres libros de $E l$ Capital.

No obstante, Paul Mattick (1974) arroja luz sobre este interrogante al decirnos que Marx (1959) conoció los planteamientos de la teoría marginal del valor, en la medida en que desplegó una precisa crítica de la teoría subjetiva de Bailey en las Teorías sobre la plusvalía.

Más aún, Marx (1882) discute a Adolf Wagner su teoría subjetiva del valor en su inédito trabajo titulado Glosas marginales al tratado de economía politica de Adolfo Wagner. Baste citar aquí el siguiente fragmento:

Después de bautizar como "valor general", como "concepto del valor", lo que solemos llamar "valor de uso", Wagner no puede por menos de recordar que el valor así (¡vaya! ¡vaya!) "derivado" ( $¡$ !) es el "valor de uso". Una vez que ha dado al "valor de uso" el nombre de "concepto del valor" en general, de "valor por antonomasia", descubre a posteriori que está charlando pura y simplemente del "valor de uso", es decir, que ha "derivado" éste, puesto que hoy el charlar y el derivar son "sustancialmente" operaciones discursivas idénticas. Pero, con este motivo, descubrimos la tramoya subjetiva que hay detrás de la anterior confusión "objetiva" de conceptos del susodicho señor Wagner. (p. 717)

La teoría del valor de Wagner, en consonancia con la teoría marginalista de Jevons, Menger y Walras, equipara el valor de uso con el valor de cambio, id est, el valor se encuentra determinado por la utilidad. O, lo que es lo mismo, la oferta y la demanda determinan el valor de las mercancías.

Podemos ir aún más lejos en la búsqueda de la crítica marxiana de la teoría utilidad marginal. Si inquirimos con mayor profundidad en el legado de Marx (1939), encontramos que en su imprescindible obra Elementos fundamentales para la crítica de la economía politica o Grundrisse, aduce que aun cuando la producción capitalista de mercancías viene a satisfacer las necesidades individuales de los consumidores, es importante comprender que la producción es inmediatamente consumo (productivo), al tiempo que el consumo es inmediatamente producción.

Mas, por otra parte, es la producción la que concibe el consumidor, en la medida en que esta modifica las necesidades de consumo y crea otras nuevas. O, dicho de otra forma: "la producción no solamente produce un objeto para el sujeto, sino también un sujeto para el objeto" (Marx, 1939, p. 13). Más pre- 
cisamente, la producción dirige el consumo por cuanto el primum movens es la acumulación del capital, esto es, la reinversión del plusvalor con el objeto de acrecentar el capital, en cuanto que valor en proceso de valorización (Astarita, 2008).

En este punto, Albert O. Hirschman (1958) y Marx se saludan cordialmente, puesto que en su Estrategia del desarrollo económico describe lo que en la economía moderna se ha venido a definir como el fenómeno de la necesidad impulsada, id est: los inventos son la madre de la necesidad.

Por su parte, Sombart (1913), desde la perspectiva de la joven escuela historicista alemana, refuerza la proposición de Marx al distinguir dos fases del capitalismoํ. En la primera, denominada capitalismo temprano, la cual comprende el período que abarca el inicio del desarrollo capitalista hasta finales del siglo XVIII, la relevancia de la figura del consumidor era tal que la concepción de que la producción de mercancías venía a satisfacer las necesidades de consumo mantenía su total vigencia.

Sin embargo, desde el siglo XIX hasta nuestros días, nace, según Sombart, un nuevo sujeto económico moderno, el gran empresario capitalista, quien tiene como máxima aspiración la obtención de la mayor ganancia posible. Por ende, busca en última instancia valorizar su capital.

Así, el interés del capitalista se centra en exclusiva en la empresa, pues el auge de la misma, escribe Sombart (1913, p. 180): "que empieza y termina siempre con una suma de dinero [D-M-...P...-M - $\left.\mathrm{M}^{\prime}-\mathrm{D}^{\prime}\right]$, está vinculado a la adquisición de un excedente [plusvalor]". Lejos queda, pues, aquel $c a$ pitalismo temprano en donde el sujeto principal era hombre real, en la medida en que éste ha sido sustituido por las abstracciones que representan la ganancia y los negocios en el capitalismo moderno. La producción sirve, pues, a la acumulación del capital, relegando la proposición marginalista y keynesiana a un estadio previo al moderno modo capitalista de producción.

Más todavía, la crítica marxiana de la teoría de la utilidad marginal puede ser descifrada en el capítulo IV del primer libro de El Capital titulado "Cómo se convierte el dinero en capital". En dicho capítulo Marx nos desvela el secreto que se oculta detrás del origen del plusvalor: la compra y venta de fuerza de trabajo. Conviene rescatar

2 En realidad, Sombart caracteriza tres fases en la historia del capitalismo en su Der Moderne Kapitalismus. Desafortunadamente, para quien escribe estas líneas, la obra nunca ha sido traducida en castellano, inglés o francés, teniendo que conformarme con otros trabajos de este autor, tales como El Burgués, donde describe las dos fases anteriormente mencionadas. 
aquí la fórmula del circuito del capital comercial:

$$
\text { D-M-D' }
$$

Esta fórmula consiste básicamente en comprar para vender más caro, lo cual reporta al capitalista una ganancia merced a la transferencia de riqueza dentro de la esfera de circulación (Shaikh, 1991, 2016). Para Keynes, Walras o Böhm-Bawerk la expresión [1] es la forma única y genuina para la obtención de la ganancia capitalista.

Sin embargo, Marx (1867) observa que en la esfera de circulación no se genera valor nuevo alguno ni tampoco mayor riqueza de la previamente existente, simplemente son distribuidos. En otras palabras, la ganancia por transferencia de riqueza implica que: "Lo que de un lado aparece como plusvalía, es del otro minusvalía; lo que de una parte representa un más, representa de la otra menos" (Marx, 1867, p. 117). No en vano es en la esfera de circulación donde las mercancías se realizan como valores y, por extensión, el plusvalor contenido en las mismas.

Así pues, cabe preguntar: ¿cuál es el secreto que ha de ser desvelado para hallar el origen del plusvalor? Hasta ahora tan solo conocemos por Marx que la fuente del valor es el trabajo humano abstracto, el cual se mide de acuerdo con el tiempo socialmente necesario. Nuestra exposición no ha sobrepasado aún los límites de la producción simple de mercancías. Hemos soslayado, por lo tanto, la clave para comprender el origen del plusvalor: la compra y venta de fuerza de trabajo.

\section{COMPRAVENTA DE LA FUER- ZA DE TRABAJO Y CARÁC- TER DUAL DEL SISTEMA DE CÁLCULO DE MARX}

En la historia universal acontece un hecho sin precedentes que constituye el punto de partida del modo capitalista de producción: la acumulación originaria. De acuerdo con Marx (1867, p. 807), la acumulación originaria: "viene a desempeñar en economía política el mismo papel que desempeña en teología el pecado original".

Esta acumulación originaria, continúa Marx (1867, p. 607), nos retrotrae a: "tiempos muy remotos [...] había de una parte, una minoría trabajadora, inteligente y sobre todo ahorrativa, y de la otra un tropel de descamisados haraganes, que derrochaban cuanto tenían y aún más".

La acumulación originaria, cual pecado original bíblico se tratase, constituye el proceso de disociación entre el obrero y la propiedad sobre las condiciones de su trabajo. Más precisamente, se trata del proceso histórico que condujo al formal divorcio entre los trabajadores y sus medios de producción. 
La teoría de la acumulación originaria de Marx identifica el rasgo más significativo del orden de sociedad capitalista, a saber, que entre los medios de producción y los trabajadores ha de mediar una separación.

Mattick (1974) recalca que aquello permitió a Marx comprender que la diferencia entre el valor de uso y el valor de cambio de la mercancía fuerza de trabajo constituye el pilar esencial en el cual descansa el modo capitalista de producción.

Es, pues, la fuerza de trabajo, y no el trabajo como habían sostenido erróneamente Adam Smith y Ricardo, la mercancía que han de vender los trabajadores a fin de poder perpetuar su existencia y la de su progenie. Los trabajadores desposeídos no solo de sus medios de producción sino también de sus medios de vida, han de ofrecer libremente en el mercado la única mercancía que poseen, su fuerza de trabajo, a cambio de un salario equivalente al valor de la cesta de bienes de consumo. El proceso, pues, de intercambio en el cual el trabajador vende su fuerza de trabajo pasa por la ulterior forma:

$$
\text { M-D-M }
$$

De la expresión [2] se sigue que el trabajador habrá de vender la mercancía fuerza de trabajo, M, a cambio de un salario, $D$, equivalente al valor de la canasta de bienes de consumo necesaria para la reproducción de su fuerza de trabajo y el mantenimiento de su familia, M.

En el otro lado de la balanza social tropezamos con el capitalista, poseedor de dinero y medios de producción, al tiempo que prisionero del impulso desenfrenado y desmesurado por obtener la máxima ganancia. Avaritia studium pecuniae habet.

El capitalista insaciable buscará en el mercado aquella peregrina mercancía que, al ser consumida, le reporte valor: la fuerza de trabajo. Nunca como en este caso puede citarse con mayor autoridad las palabras de Virgilio: auri sacra fames.

El trabajador, desposeído y a la vez libre para decidir ser explotado, habrá de vender, pues, su fuerza de trabajo temporalmente. De lo contrario, dice Marx (1867, p. 121): "si la vende en bloque y para siempre, lo que hace es venderse a sí mismo, convertirse de libre en esclavo, de poseedor de una mercancía en mercancía".

Para desvelar el secreto que se esconde detrás del plusvalor, hemos de desglosar el esquema del circuito del capital industrial:

$$
\text { D-M \{FT/MP...P...M'-D' }
$$

En conformidad con el esquema [3] se nos aparecen dos intercambios 
de equivalentes del cual no podrá en modo alguno dimanar el plusvalor, a saber: 1) $\mathrm{D}=\mathrm{M}$; y 2) $\mathrm{M}^{\prime}=\mathrm{D}^{\prime}$. De ello se infiere que el incremento de valor ha de haberse realizado durante la fase productiva, (...P...). Debe advertirse, empero, que el aumento de valor no podrá corresponder, en forma alguna, a los medios de producción, en la medida en que estos, en cuanto trabajo muerto u objetivado, tan sólo transfieren el valor que contienen nacido de un trabajo pretérito.

Así pues, el valor generado en el proceso de producción habrá de derivar, stricto sensu, del valor agregado por el trabajo vivo, esto es, por la fuerza de trabajo. En otras palabras, el capitalista habrá recibido un valor producido por la fuerza de trabajo por el cual no habrá entregado equivalente alguno.

Puesto que el salario que perciben los trabajadores equivale al valor de la cesta de bienes de consumo, el valor agregado o valor añadido es aquel que se compone, de una parte, de tiempo de trabajo necesario para producir la cantidad de mercancías que permiten reproducir la fuerza de trabajo. De otra, se trata de trabajo excedente o trabajo no retribuido que encarna el plusproducto o plusvalor apropiado por el capitalista en virtud del derecho que ejerce sobre la propiedad privada de los medios de producción.
Henos aquí ante el fin del misterio del plusvalor. Los capitalistas, quienes detentan el control sobre el proceso laboral, poseen la capacidad o habilidad de extender o intensificar la jornada laboral por encima del tiempo de trabajo necesario para producir la cantidad de mercancías que consienten el sustento vital del trabajador y el de su familia.

$\mathrm{O}$, más exactamente, el secreto del plusvalor consiste en que el salario real ha de intercambiarse a razón de una tasa inferior a la de la productividad del trabajo. Se deprende, por ende, que la ganancia del capital, id est, el plusvalor realizado en la esfera de circulación, nace de la explotación de la fuerza de trabajo.

No obstante, Grossmann (1929) remarca que la especificidad de la producción capitalista de mercancías no solo se revela por medio del proceso laboral, en el cual, baste decir, la fuerza de trabajo y los medios de producción engendran conjuntamente el producto. Existe un principio dual, añade Grossman. Por un lado, se trata de un proceso laboral cuyo fin es crear un producto. Por el otro, involucra un proceso de valorización.

El capital es, en efecto, valor en proceso de valorización, por cuanto los medios de producción y la fuerza de trabajo son asimismo valores: capital constante, c, y capital variable, $v$, respectivamente. Ello nos remite a 
la formulación clásica del valor de la mercancía de Marx:

$$
\mathrm{V}=\mathrm{c}+v+p
$$

Donde denota el valor de los medios de producción consumidos en un período, en tanto que $v+p$ define el valor agregado. Centremos ahora nuestra atención en cada uno de los componentes del valor de la mercancía. Comenzando por el capital constante, $c$, este se descompone en dos partes: i) capital ijo; y ii) capital circulante. A este respecto, Schumpeter (1942, 1954) elogia el gran acierto de Marx al reemplazar la caduca distinción legada por Ricardo, puesto que el economista clásico incluyó, no sin antes incurrir en error, el fondo de salarios como parte del capital circulante.

En Marx, por el contrario, el capital circulante corresponde a las materias primas, bienes intermedios u otros insumos cuyo tiempo de rotación es igual o inferior a un año. Esto es, se trata de elementos de capital constante cuyo consumo productivo podrá durar escasos meses y, por consiguiente, rotarán varias veces a lo largo de un año. Por su parte, los elementos de capital fijo, tales como la maquinaria, los equipos o las plantas, servirán a la producción durante un lapso que podrá superar los 20 o 30 años (Lange, 1965).

Baste señalar aquí que Marx (1885, pp. 159-220), en el libro II de El Capital, introdujo el concepto de rotación del capital constante. De acuerdo con Oskar Lange (1965, p. 29), dicho concepto se define como: "el promedio de duración de los medios de producción en el proceso productivo, y por consiguiente se trata aquí de la duración económica media, y no necesariamente del desgaste".

Así, si denotamos la rotación del capital constante con el símbolo, tenemos que su inversa corresponde al índice de reposición $\mu$ :

$$
1 / \tau=\mu
$$

Por lo tanto, el stock de capital constante, $K$, en adelante, multiplicado por el índice de reposición corresponderá al valor del flujo de los medios de producción consumidos durante un período, c:

$$
\mathrm{c}=K \mu=K 1 / \tau
$$

$\mathrm{O}$, lo que es lo mismo:

$$
K=\mathrm{c} \tau
$$

En cuanto al stock de capital variable, en adelante, $\mathrm{Z}$, es importante distinguirlo del fondo salarial, $v$. Cabe subrayar que el capital variable solamente podrá ser igual al fondo salarial cuando la remuneración de los asalariados se reduzca a un único año. En consonancia con lo anterior, el capital variable habrá de ser igual al fondo 
de salarios multiplicado por el período de rotación $\theta$ :

$$
\mathrm{Z}=v \theta
$$

Este inciso nos permitirá diferenciar los conceptos de composición técnica de capital, composición de valor del capital y composición orgánica de capital.

Comenzando por la composición técnica de capital, esta hace referencia a la relación entre la cantidad en términos físicos de medios de producción (MP) y la cantidad en términos físicos de fuerza de trabajo (FT) puesta en marcha por los capitalistas en el proceso productivo, es decir, MP: FT.

Por su parte, la composición de valor de capital o composición orgánica de los inputs representa la relación entre el flujo del valor de los medios de producción consumidos durante un año y el fondo salarial (Lange, 1965):

$$
k=c / v
$$

Finalmente, la composición orgánica de capital expresa el cociente entre el stock de capital constante y el stock de capital variable:

$$
K / Z=c \tau / v \theta=k \cdot \tau / \theta \quad[10]
$$

Pasemos, pues, al último componente del valor de las mercancías: el plusvalor. Como se subrayó previamente, el plusvalor nace merced a la habi- lidad del capitalista para prolongar o intensificar la jornada laboral más allá del tiempo necesario para que un trabajador produzca la cantidad de mercancías que aseguren su existencia y la de su familia. Lo que para el trabajador es plustrabajo, para el capitalista es un plusvalor compuesto de: i) ganancias; ii) intereses; iii) rentas; iv) e impuestos.

Nótese, por una parte, que al realizarse el plusvalor en la esfera de circulación, la totalidad del mismo no va a parar a los capitalistas en forma de ganancia, en la medida en que el propietario de los medios de producción ha de hacer frente a sus obligaciones con otros agentes económicos: el banco o prestamista privado (intereses), propietario de la tierra (renta) y el Estado (impuestos).

Por otra, el valor agregado encarna la totalidad del trabajo ejecutado durante un período, id est, el trabajo retribuido (capital variable) más el trabajo no retribuido (plusvalor):

$$
\text { valor agregado }=v+p
$$

Es de destacar que en el capítulo VII del libro I de El Capital, Marx deriva de la expresión [11] la ecuación de la tasa de plusvalor o tasa de explotación de la fuerza de trabajo, siendo la misma la relación del plusvalor entre el capital variable, (en adelante, e): 


$$
e=p / v
$$

Mientras que de la relación entre el plusvalor, capital constante y el capital variable (la composición orgánica de capital), Marx infiere su ecuación de la tasa de ganancia. En este sentido, considérese, primeramente, que la tasa de ganancia se expresa como el cociente del plusvalor entre el sumatorio de los stocks de capital constante y capital variable, obteniendo así:

$$
B / K=p /(K+Z)
$$

En segundo término, si descomponemos la ecuación [13] tenemos:

$$
\begin{gathered}
B / K=p /(K+Z)=(p / v) /(c \tau / v+\theta)= \\
p /(k \tau / \theta+1) \theta \quad[14]
\end{gathered}
$$

Puesto que:

$$
K / Z=c \tau / v \theta=k . \tau / \theta
$$

La tasa de ganancia habrá de ser:

$$
B / K=p /(K / Z+1) \theta
$$

En conformidad con Marx, la tasa de acumulación, esto es, la relación entre la inversión productiva y el plusvalor, habrá de encontrarse estrechamente relacionada con la tasa de ganancia.

Esto quiere decir que las decisiones de inversión de los capitalistas tienen como estímulo principal la evolución de la tasa de ganancia realizada y esperada. A fin de comprender tal relación, consideraremos por caso una economía dividida en $n$ sectores, en tanto que el producto global del $i$-enésimo sector se denota de acuerdo con la siguiente expresión:

$$
X_{i}=a_{0 i} X_{i}+\sum_{j=1}^{n} a_{i j} X_{i}+p_{i}
$$

Donde $a_{0 i}$ y $a_{i j}$ se refieren, en sentido de Leontief (1951), a los coeficientes de empleo y los coeficientes técnicos, respectivamente. Consecuentemente, el plusvalor que se apropian los capitalistas del sector $i$ será igual a:

$$
p_{i}=X_{i}\left(1-a_{0 i}-\sum_{j=1}^{n} a_{i j} X_{i}\right)
$$

Según Lange (1965), el nexo existente entre la inversión productiva y la tasa de ganancia podrá derivarse de la siguiente ecuación:

$$
X_{i}-x_{i}^{(0)}-\sum_{j=1}^{n} a_{i j} X_{j}=\alpha_{i} X_{i}-\sum_{j=1}^{n} a_{i j} X_{j}
$$

En la formulación [19], $\alpha_{i}$ expresa la tasa de inversión bruta, asimismo, nos indica que la inversión neta (inversión para la ampliación) ha de ser proporcional a la tasa de ganancia obtenida por el capital constante producido en dicho sector, esto es, $\frac{p_{i}}{K_{i}}$. Por tanto, se tiene: 


$$
\alpha_{i} X_{i}-\sum_{j=1}^{n} a_{i j} X_{j}=\gamma_{i} \frac{p_{i}}{K_{i}}
$$

Siendo $\gamma_{i}>0$ el coeficiente de proporcionalidad. Ahora bien, Lange (1964) advierte que la expresión [20] puede emplazarnos a innecesarias complicaciones contables, lo cual obliga a transformar la inversión neta en una función lineal dependiente del plusvalor y el capital constante:

$$
\alpha_{i} X_{i}-\sum_{j=1}^{n} a_{i j} X_{j}=\gamma_{i} p_{i}-g_{i} K_{i}
$$

En este caso se tiene que $\gamma_{i}>0$ y $g_{i}>0$ corresponden al coeficiente de propensión a la inversión ante las expectativas de beneficios y el coeficiente de sensibilidad de la inversión ante las variaciones del capital constante, respectivamente.

En otras palabras, si el coeficiente de propensión a la inversión ante las expectativas de beneficios es alto, ceteris paribus, la inversión neta será tanto mayor cuanto mayor sea el plusvalor apropiado por los capitalistas.

Por otro lado, si el coeficiente de sensibilidad de la inversión ante las variaciones del capital constante es elevado, ceteris paribus, la inversión neta será tanto menor cuanto mayor es el crecimiento del stock capital constante.
Así pues, el incremento simultáneo de la magnitud de plusvalor y el stock de capital constante hará aumentar o disminuir la inversión neta dependiendo de cuál de los dos coeficientes es mayor.

Cabe reseñar que la ecuación [21] es muy similar a la expresión matemática que llega Kalecki (1939, p. 31) en el capítulo I de su obra Estudios sobre la teoría de los ciclos económicos. Al igual que Marx y Lange, el economista polaco determina que el volumen de inversiones: "es una función creciente de la acumulación bruta y una función decreciente del volumen del equipo de capital K'.

Tras este inciso ha de advertirse que a partir de este punto el sistema marxiano se desdobla en dos métodos de cálculo. i) La ecuación [12] de la tasa de explotación corresponde al sistema de cálculo de valores. ii) En tanto que la expresión [16] referente a la tasa de ganancia se reserva al sistema de cálculo de precios de producción. Más exactamente, bajo un régimen capitalista de producción de mercancías, la separación entre productores y medios de producción conlleva la explotación de la fuerza de trabajo. Por tanto, valores y precios no podrán ser en modo alguno proporcionales (Marx, 1894). Baste señalar aquí que dicho carácter dual del modelo marxiano nunca ha estado exento de polémica, pues hasta 
nuestros días sigue arrojando extensos ríos de tinta.

Fue Böhm-Bawerk, no obstante, el representante más notorio de una extensa estirpe de formidables adversarios de Marx que han creído haber hallado una contradicción irresoluble entre el libro I y el libro III de El Capital.

En su trabajo titulado La conclusión del sistema marxiano, Böhm-Bawerk (1896) sostiene que en tanto en el libro I las mercancías se intercambian con arreglo a la cantidad de trabajo incor-porado en las mismas, en el libro III Marx con harta frialdad y precisión, pasa a considerar que las mercancías se venden de acuerdo con sus precios.

Tal es el desconcierto del economista austriaco que no puede por menos que confesar a sus lectores: "Yo no sé qué hacer, pues no veo aquí en absoluto la explicación y el ajuste de un problema controvertido, veo aquí sólo una pura y simple contradicción. El tercer volumen de Marx desmiente al primero" (Böhm-Bawerk, 1896, p. 49). Nada podría ser más erróneo. Marx (1939, p. 62) ya escribía en sus Grundrisse que valores y precios, en manera alguna, serían proporcionales.

Schumpeter (1954, p. 663) lanza esta misma objeción, puesto que: “[...] incluso el más grande de los críticos de Marx durante el siglo XIX, BöhmBawerk, pensó que Marx expuso una teoría del valor cantidad de trabajo en el primer volumen de Das Kapital, [...] Pero esa interpretación es un error, error que implica no haber visto el punto esencial de la teoría marxiana del valor". Conviene destacar aquí que el objeto de Marx a lo largo de la elaboración de los tres libros que conforman El Capital fue analizar la transformación de los valores en precios de producción.

En el libro I, los valores de las mercancías son proporcionales a los precios directos como consecuencia de la total uniformidad de la composición orgánica de capital. En el libro II, Marx transita desde un modelo unisectorial hacia otro bisectorial, donde las composiciones orgánicas de capital del sector I y el sector II difirieren como consecuencia de la especialización productiva de cada uno de ellos. El sector I es intensivo en capital constante, en tanto que el sector II lo es en fuerza de trabajo.

Consecuentemente, el dual del modelo del libro II medido en precios de producción lo encontramos recogido en el libro III, donde el filósofo de Tréveris elucida la transformación de valores en los precios de producción de las mercancías. Vale decir que dichos precios de producción garantizan una rentabilidad media (tasa de ganancia promedio) para cada una de las ramas de la producción que componen una 
economía capitalista (Boundi Chraki, 2014). Este inciso, en cuanto a la di-ferencia entre valores y precios, no ha sido ocioso, en la medida en que nos sirve para introducirnos a continuación en la comprensión del teorema marxiano fundamental.

\section{EL TEOREMA MARXIANO FUNDAMENTAL}

Varios economistas han tenido la particular virtud de confundir los conceptos de tasa de explotación y de tasa de ganancia de Marx. Sírvase a título de ejemplo la extraña aserción de Joan Robinson (1942, p. 36), según la cual la relación entre tasa de plusvalor y la tasa de ganancia no es más que un razonamiento tautológico.

Morishima (1973) discrepa de la afirmación de Robinson, puesto que la tasa de plusvalor pertenece al sistema de cálculo en términos de valores, en tanto que la tasa de ganancia se reserva al cálculo en términos de precios de producción. La relación entre ambas no se trata en modo alguno de una tautología. Rosdolsky (1968), por su parte, es menos condescendiente con Joan Robinson a este respecto.

Rosdolsky hace notar que la teoría de Marx no se basa en forma alguna en el supuesto de una tasa constante de plusvalor, tal y como parece deducir Robinson. Este error, arguye Rosdolsky, nace merced a la incapacidad de Robinson por comprender que el capital es una relación social y, en cuanto tal, se trata de una categoría sociohistórica.

Retomando la relación entre valores y precios, baste señalar que ello constituye el pilar sobre el que se edifica el teorema fundamental marxiano. $\mathrm{O}$, lo que es los mismo, el modelo Okishio-Morishima-Seton (Okishio, 1963; Morishima \& Seton, 1961; Morishima, 1973, 1974). Pasemos a discernir dicho teorema. Morishima (1973) señala que en el libro I de El Capital se recogen tres definiciones alternativas de la tasa de plusvalor, o tasa de explotación.

La primera de dichas definiciones se deriva del vector que recoge la cantidad de bienes salario necesarios para la reproducción de la fuerza de trabajo:

$$
B=\left[\begin{array}{l}
b_{n+1} \\
\cdots \\
b_{m}
\end{array}\right]
$$

Considérese $\bar{T}$ y T como la longitud máxima y dominante de la jornada laboral, respectivamente. De todo lo anterior, se infiere que los bienes salario necesarios para la reproducción de la fuerza de trabajo medidos en términos de tiempo de trabajo se valoran a razón de $\lambda_{I I} B$; siendo $\lambda_{I I}$ los bienes salarios producidos por el sector II de bienes de consumo y $B$ la cantidad necesaria 
de los mismos. De ello se desprende la siguiente desigualdad:

$$
T>\lambda_{I I} B
$$

De acuerdo con la expresión [23], un asalariado podrá trabajar al día un número total de horas superior a las necesarias para producir las mercancías equivalentes a sus medios de vida. Debe recalcarse que esta hipótesis es condición sine qua non, en la medida en que: "de otro modo, el trabajador sólo podría producir, en el mejor de los casos, las mercancías necesarias para su subsistencia diaria" (Morishima, 1973, p. 61). En otras palabras, la hipótesis básica de la teoría de la explotación ha de cumplir con:

$$
\bar{T} \geq T>\lambda_{I I} B
$$

Consiguientemente, el precio mínimo de la mercancía fuerza de trabajo se situará en un nivel equivalente al valor de los bienes salario, esto es, $\lambda_{I I} B$. Las horas necesarias para reproducir el valor equivalente de los bienes salario pasa a definirse como $\mathrm{T}$.

Considerando $w=1 / \mathrm{T}$, el trabajador percibirá $w$ unidades de bienes salario al día y por hora a razón de una oferta de una unidad de fuerza de trabajo por hora. Por lo tanto, la suma de $w B$ equivale a $w \lambda_{I I} B$ horas de trabajo. De esta suerte, $w \lambda_{I I} B$ representará la parte retribuida, en tanto que 1- $w \lambda_{I I} B$, la parte no retribuida, id est, el plustrabajo.
Más exactamente, el salario percibido por los trabajadores corresponde al coste de producción de la fuerza de trabajo. En este sentido, el grado de explotación, $e$, se expresará como el cociente entre el trabajo no retribuido y el trabajo retribuido:

$$
e=\frac{\text { trabajo no retribuido }}{\text { trabajo retibuido }}=\frac{1-w \lambda I I B}{w \lambda I I B}
$$

La ecuación [25] es, en definitiva, la primera definición de la tasa de explotación. Para la segunda definición, es importante subrayar que en el libro I de El Capital Marx denomina alternativamente el trabajo no retribuido y el trabajo retribuido como trabajo excedente y trabajo necesario, respectivamente.

El trabajo necesario pertenece a la parte de la jornada laboral en la cual el trabajador reproduce el valor equivalente a los bienes salario. El trabajo excedente, por su parte, se refiere al valor producido por el que el trabajador no percibe equivalente alguno (Marx, 1867, p. 165).

Así pues, el grado de explotación podrá expresarse como el cociente del trabajo excedente entre el trabajo necesario:

$$
e=\frac{\text { Trabajo excedente }}{\text { Trabajo necesario }}
$$

Análogamente, el grado de explotación podrá ser definido de acuerdo con 
la distribución sectorial del trabajo. En primer término, bajo el supuesto de que en una sociedad con $\bar{N}$ trabajadores con una jornada laboral de $T$ horas diarias, se considera que los trabajadores producen una cantidad diaria de $B N$ bienes salario para cubrir sus necesidades fisiológicas. Esto quiere decir que deberán producir diariamente una cantidad de $A_{I I} B N$ de bienes de capital para el sector II de bienes de consumo.

En segundo término, la demanda de bienes de capital por parte del sector II de bienes de consumo repercutirá en el sector I productor de medios de producción. Merced a ello se genera un efecto multiplicador que trasciende en la cantidad de bienes de capital producidos por el sector I:

$$
\overline{x_{I}}=A_{I} \bar{x}_{I}+A_{I I} B \bar{N}
$$

Por lo tanto, los requerimientos de trabajo directo e indirecto necesarios para producir las cantidades de bienes salarios demandas habrá de satisfacer la siguiente ecuación:

$$
T N=L_{I} \bar{x}_{I}+L_{I I} B \bar{N}
$$

Donde $\bar{N}$ es el total de trabajadores necesarios, mientras que $\bar{N}-N$ es el excedente de oferta de fuerza de trabajo que puede trabajar, indistintamente, en el sector I de medios de producción y el sector $\mathrm{II}_{b}$ de bienes de lujo para capitalistas. Dicho excedente, vale decir, actuará como un ejército industrial de reserva cuya función es evitar que los aumentos salariales absorban las ganancias de los capitalistas (Marx, 1867).

Más aún, la existencia de un ejército industrial de reserva es una condición sine qua non para el sostenimiento del modo capitalista de producción, tanto más cuanto que debe existir una oferta relativa de fuerza de trabajo ilimitada que satisfaga la demanda creciente del capital durante el proceso de acumulación de capital (Marx, 1885).

De las ecuaciones [27] y [28] se infiere que el cociente del trabajo excedente total entre el trabajo socialmente necesario habrá de ser igual al grado de explotación de la fuerza de trabajo:

$$
\frac{T \bar{N}-T N}{T N}=\frac{T N-\left[L_{I}\left(I-A_{I}\right)^{-1} A_{I I}+L_{I I}\right] B N}{\left[L_{I}\left(I-A_{I}\right)^{-1} A_{I I}+L_{I I}\right] B N}=\frac{1-w \lambda I I B}{w \lambda I I B}
$$

Por ende:

$$
e=\frac{\text { Trabajo excedente total }}{\text { Trabajo socialmente necesario }}=\frac{T \bar{N}-T N}{T N}
$$


Es de hacer notar que es factible expresar el grado de explotación como el cociente del total del plusvalor producido entre el total del valor de la fuerza de trabajo. Dicho en otros términos, se trata de la tercera y última definición de la tasa de explotación que colige Morishima (1973) de El Capital de Marx.

En este caso, si tomamos $x_{I}$ y $x_{I I}$ como vectores de producción de los sectores de medios de producción y bienes salarios, respectivamente, podemos expresar el empleo total como:

$$
T \bar{N}=L_{I} x_{I}+L_{I I} x_{I I}
$$

De la ecuación [31] se deduce que el sector II de bienes salario deberá producir una cantidad de $B N$ para satisfacer las demandas de bienes de consumo de $\operatorname{los} N$ trabajadores. Asimismo, para que el sector I y el sector II pue- dan producir $x_{I}$ y $x_{I I}$, respectivamente, el sector I habrá de producir una cantidad de bienes de capital a razón de:

$$
x_{I}^{*}=A_{I} x_{I}+A_{I I} x_{I I}
$$

En conformidad con la ecuación [32], el producto excedente del sector I ha de ser $x_{I}-x_{I}^{*}$. Así, el producto excedente del sector II de bienes salario vendrá dado por $x_{I I}-B \bar{N}$.

En consecuencia, el total del plusvalor producido, $s$, será:

$$
s=\lambda_{I}\left(x_{I}-x_{I}^{*}\right)+\lambda_{I I}\left(x_{I I}-B \bar{N}\right)[33]
$$

Mientras que el valor de la fuerza de trabajo equivale a $\lambda_{I I} B \bar{N}$.

Es factible, por otro lado, expresar la tasa de plusvalor, s', como el cociente del plusvalor entre el valor total de la fuerza de trabajo:

$$
s^{\prime}=\frac{\text { Plusvalor }}{\text { Valor total de la fuerza de trabajo }}=\frac{\lambda_{I}\left(x_{I}-x_{I}^{*}\right)+\lambda_{I I}\left(x_{I I}-B \bar{N}\right)}{\lambda_{I I} B \bar{N}}
$$

Según la teoría Marx, la tasa de plusvalor ha de ser igual al grado de explotación de la fuerza de trabajo. Si expresamos el valor total de la fuerza de trabajo obtenemos:

$$
\begin{gathered}
\lambda_{I I} B \bar{N}=w \lambda I I B\left(L_{I} x_{I}+L_{I I} x_{I I}\right)[35] \\
\lambda_{I}\left(x_{I}-x_{I}^{*}\right)+\lambda_{I I}\left(x_{I I}-B \bar{N}\right)=\left(\lambda_{I}-\lambda_{I} A_{I}-w \lambda_{I I} B L_{I}\right) x_{I}+\left(\lambda_{I I}-\lambda_{I} A_{I I}-w \lambda_{I I} B L_{I I}\right) x_{I I}
\end{gathered}
$$


A partir de la ecuación [25] se infiere que:

$$
(1+e) w \lambda_{I I} B=1
$$

De la expresión [37] obtenemos las ecuaciones de determinación de los valores:

$$
\begin{aligned}
& \lambda_{I}=\lambda_{I} A_{I}+w \lambda_{I I} B L_{I}+e w \lambda_{I} B L_{I} \\
& \lambda_{I I}=\lambda_{I} A_{I I}+w \lambda_{I I} B L_{I I}+e w \lambda_{I I} B L_{I I}
\end{aligned}
$$

Con las ecuaciones [38] y [39] es factible expresar los paréntesis del segundo miembro de la ecuación [36] como ew $\lambda_{I I} B L_{I}$ y $e w \lambda_{I I} B L_{I I}$, respectivamente.

De ello se desprende:

$$
\begin{gathered}
\lambda_{I}\left(x_{I}-x_{I}^{*}\right)+\lambda_{I I}\left(x_{I I}-B \bar{N}\right) \\
=e w \lambda_{I I} B\left(L_{I} x_{I}+L_{I I} x_{I I}\right)
\end{gathered}
$$

Por tanto, la tasa de plusvalor es igual al grado de explotación. Finalmente, llegamos a las siguientes igualdades:

$\frac{\text { Plusvalor }}{\text { (Valor de la fuerza de trabajo) }}$
$=\frac{(\text { Trabajo excedente) }}{(\text { Trabajo necesario) }}$
$=\frac{(\text { Trabajo retribuido) }}{(\text { Trabajo no retribuido) }[41]}$

Tras demostrar que la tasa de plusvalor equivale al grado de explotación, debe indicarse que el primer término del segundo miembro de cada ecuación representa el valor de los medios de producción utilizados por la $i$-enésima industria.

A este respecto, recuérdese que dicho valor es designado por Marx como el capital constante (capital fijo más capital circulante). El segundo término designa el valor de la fuerza empleada por la $i$-enésima industria, esto es, el capital variable. El tercer término, en cuanto tiempo de trabajo excedente, es el plusvalor producido en la $i$-enésima industria y apropiado por los capitalistas de dicho sector. En consecuencia, el producto total de la $i$-enésima industria es la suma total del capital constante, $c_{i}$, más el capital variable, $v_{i}$, más el plusvalor, $p_{i}$ :

$$
\lambda_{i}=c_{i}+v_{i}+p_{i},(i=1, \ldots, n)
$$

La tasa de plusvalor de la $i$-enésima industria es igual a $e$ para todo valor de $i$. O, más exactamente, el grado de explotación es uniforme en toda la economía. Esto quiere decir que la longitud de la jornada laboral es la misma en toda la economía en virtud de la definición del grado de explotación, $e$. Más precisamente, bajo el supuesto de que la longitud de la jornada laboral difiere en dos industrias cualesquiera, en adelante, $i$ y $j$, los grados de explotación de ambas podrá expresarse como: 


$$
\begin{gathered}
e_{i}=\frac{T_{i}-\lambda_{I I} B}{\lambda_{I I} B} \\
e_{j}=\frac{T_{j}-\lambda_{I I} B}{\lambda_{I I} B}
\end{gathered}
$$

Si $\mathrm{T}_{\mathrm{i}} \neq \mathrm{T}_{\mathrm{j}}$, ambas expresiones de los grados de explotación deben diferir, en tanto que si $\mathrm{T}_{\mathrm{i}}>\mathrm{T}_{\mathrm{j}}$, se colige que la industria $j$ se muestra más atractiva y ventajosa para el trabajador del sector $i$.

Aquello conducirá a un trasvase de fuerza de trabajo de $i$ a $j$ hasta alcanzar el equilibrio de $\mathrm{T}_{\mathrm{i}}=\mathrm{T}_{\mathrm{j}}$. Según Marx (1894), la nivelación en el grado de explotación nace merced a la concurrencia entre obreros a través de la emigración de una rama de producción a otra.

Llegados a este punto, es momento de pasar a examinar el enunciado marxiano de que el plusvalor es la fuente misma de la ganancia capitalista. A partir de aquí es imperativo abandonar el sistema de cálculo de valores para introducirnos en el sistema de cálculo de precios. En primer lugar, si consideramos como el precio de la $i$-enésima mercancía obtenemos los siguientes vectores de precios de los medios de producción y de bienes salario:

$$
\begin{aligned}
& p_{I}=\left(p_{i}, \ldots, p_{n}\right) \\
& p_{I I}=\left(p_{n+1}, \ldots, p_{m}\right)
\end{aligned}
$$

En segundo lugar, se considera que la tasa salarial, $w$, se sitúa al nivel de subsistencia. Por ende, el trabajador podrá adquirir una cantidad $w B$ de bienes salario equivalentes al salario monetario recibido por hora trabajada. De este modo, tenemos:

$$
w \geq p_{I I} w B
$$

Según Morishima (1973, p. 65), a partir de la desigualdad de la ecuación [47] se deduce la hipótesis (1) de Marx de que las mercancías se venden con arreglo a su valor, a saber: "los precios pueden ser normalizados de modo que $\lambda_{I I}=p_{I I} \quad B$, con la consecuencia de que $\lambda_{I I} B=p_{I I} B$.

Por su parte, la hipótesis (2) de Marx implica que $W \geq p_{I I} B$, donde $W$ es el precio de la fuerza de trabajo total. De este modo, dividiendo ambos términos de la desigualdad entre $T$, y denominando $w$ como tasa salarial/hora, esto es, W/T, se obtiene la expresión [47]. Por consiguiente, los capitalistas de todos los sectores obtendrán ganancias positivas siempre y cuando se satisfagan las siguientes desigualdades:

$$
\begin{aligned}
& p_{I}>p_{I} A_{I}+w L_{\mathrm{I}} \\
& p_{I I}>p_{I} A_{I I}+w L_{I I}
\end{aligned}
$$

Cabe ahora preguntar: ¿cuáles son las condiciones necesarias y suficientes para que exista un conjunto de precios $\mathrm{y}$ tasas no negativas que deriven en ganancias positivas para todas las ramas de la producción? 
La respuesta a dicha cuestión fue dada por Okishio (1963). Según Okishio, para que exista un conjunto de precios y una tasa salarial que satisfaga las desigualdades [48] y [49] es necesario $y$ suficiente que exista una tasa de salarios reales, $w$, que consienta un grado de explotación, $e$, positivo.

En otras palabras, si la tasa de salarios reales es inferior a la productividad del trabajo, el grado de explotación será positivo. He aquí, pues, la condición necesaria y suficiente para el mantenimiento del orden de sociedad capitalista.

Procedamos a la demostración de la mentada condición necesaria y suficiente. En primer lugar, hemos de suponer que las desigualdades de [48] y [49] se cumplen, asimismo, tenemos que $e>0$. En segundo lugar, hemos de sustituir en [48] y [49] $w$ por su valor en la expresión [47], obteniendo en este caso:

$$
\begin{aligned}
& p_{I}>p_{I} A_{I}+p_{I I} w B L_{I} \\
& p_{I I}>p_{I} A_{I}+p_{I I} w B L_{I I}
\end{aligned}
$$

De ello se deduce la matriz de coeficientes de capital y coeficientes técnicos de medios de vida para los trabajadores:

$$
\left[\begin{array}{cc}
A_{I} & A_{I I} \\
w B L_{I} & w B L_{I I}
\end{array}\right]
$$

La matriz es productiva por cuanto $p_{I} \mathrm{y}$ $p_{I I}$ son positivos. Ello implica que los vectores de producción son positivos:

$$
\left[\begin{array}{c}
x_{I} \\
x_{I I}
\end{array}\right]>\left[\begin{array}{cc}
A_{I} & A_{I I} \\
w B L_{I} & w B L_{I I}
\end{array}\right]\left[\begin{array}{l}
x_{I} \\
x_{I I}
\end{array}\right]
$$

Premultiplicando [53] por el vector positivo $\left(\lambda_{P}, \lambda_{I I}\right)$ y considerando [38] y [39], se obtiene:

$$
\begin{gathered}
\left(\lambda_{I} x_{I}+\lambda_{I I} x_{I I}\right)-\lambda_{I}\left(A_{I} x_{I}+A_{I I} x_{I I}\right)-\lambda_{I I}\left(w B L_{I} x_{I}+w B L_{I I} x_{I I}\right) \\
=e\left(w \lambda_{I I} B L_{I} x_{I}+w \lambda_{I I} B L_{I} x_{I}\right)>0
\end{gathered}
$$

Se demuestra de la ecuación [54] que $e$ es positivo.

A la inversa también podemos demostrar el teorema fundamental marxiano. Es decir, en presencia de explotación es posible que todos los ramos de producción obtengan beneficios positivos. Dado $e>0$, se infiere de [38] y [39] que:

$$
\begin{aligned}
& \lambda_{I}>\lambda_{I} A_{I}+\lambda_{I I} w B L_{I} \\
& \lambda_{I I}>\lambda_{I} A_{I I}+\lambda_{I I} w B L_{I}
\end{aligned}
$$

Donde:

$$
\begin{gathered}
p_{I}=\alpha \lambda_{I} \\
p_{I I}=\alpha \lambda_{I I} \\
w=\alpha \lambda_{I I} w B
\end{gathered}
$$


Siendo cualquier número positivo, se demuestra que todos los términos son positivos y satisfacen las condiciones de [43] y [44]. Por tanto, el modelo Okishio-Morishima-Seton, en cuanto teorema fundamental marxiano, tiene la virtud de ser el entrelazo que une el sistema de valores expresado en las ecuaciones [38] y [39] y el sistema de precios de las expresiones [50] y [51] propios de la teoría de Marx. Consecuentemente, en presencia de ganancias positivas, $e$ será positivo. $\mathrm{O}$, más exactamente, los trabajadores recibirán un salario inferior al valor que producen. Grosso modo, las condiciones necesarias para que la tasa de explotación sea positiva son:

1) La tecnología en uso ha de permitir que los medios de producción sean productivos, pues de lo contrario se obtendrán valores negativos.

2) Las técnicas adoptadas deben consentir que el valor de los bienes salarios sea inferior a la jornada de trabajo máxima.

3) La jornada efectiva de trabajo ha de ser superior al tiempo de trabajo necesario, esto es, la tasa salarial debe ser menor que la productividad del trabajo.

Llegados a este punto, no podemos pasar por alto el extraño argumento de John Roemer (1986) con respecto a la explotación, el valor y el precio de las mercancías. En su Valor, explotación y clase, Roemer (1986, p. 50) sostiene que el problema de la explotación es independiente de la relación entre valores y precios, siendo ello, por tanto, un anacronismo.

Según este autor, el trabajo abstracto, en cuanto fuente del valor, no puede anteceder a los precios, por cuanto es el precio el que determina el valor, no su opuesto, como expuso brillantemente Marx en El Capital. Pero esto no es todo. Roemer (1986, p. 65), siguiendo los postulados neomarxistas y dependentistas (Baran, 1957; Baran \& Sweezy, 1966; Gunder-Frank, 1966; Emmanuel, 1970; Amin, 1973) sobre el intercambio desigual, sostiene que la explotación de trabajo es, en realidad, una transferencia de valor de los explotados a los explotadores.

Más concretamente, los explotados poseen una baja relación capital/trabajo, en tanto que los explotadores se caracterizan por una elevada relación capital/trabajo. De esta suerte, el problema de la explotación se localiza en la esfera de circulación y no, como demuestra Marx, en la esfera de producción.

Roemer concluye, por tanto, que la explotación es per de initionem un intercambio desigual del trabajo, en virtud de lo cual las relaciones de dominación nada tienen que ver con dicha cuestión. Roemer (1986, p. 97) retrocede así a los tiempos de Proud'hon al considerar la explotación como una injusticia social, id est: "como la naturaleza injusta de los flujos que resultan 
de una distribución injusta de los activos iniciales".

Retomando, pues, la explicación del teorema marxiano fundamental cabe reseñar que el grado de explotación de la fuerza de trabajo estará sujeto tanto a la duración de la jornada laboral como a la intensidad de la misma. O, para ser más exactos, los capitalistas, en función de la debilidad o fortaleza de los trabajadores, podrán elevar la tasa de explotación modificando la proporción del trabajo excedente y el trabajo necesario a lo largo de una jornada normal de trabajo. Por lo pronto, esto implica dos mecanismos que podrán ensanchar la producción de plusvalor apropiado por los capitalistas.

El primero consiste en alargar la jornada laboral manteniendo constante el trabajo necesario, lo cual elevará el trabajo excedente al mismo tiempo que mantiene inalterado el trabajo necesario. Marx (1867) denominó este mecanismo como plusvalor absoluto.

El segundo mecanismo consiste en incrementar la capacidad productiva de la fuerza de trabajo, reduciendo con ello la proporción del tiempo necesario a través del abaratamiento de los medios de vida. Esta modalidad implica el acrecentamiento de la intensidad normal del trabajo, la cual en sentido de Marx (1867), se define como el plusvalor relativo.

Esto es todo cuanto hemos de decir acerca de la teoría del plusvalor de
Marx. Cabe señalar que la teoría marxiana de la explotación conmina a comprender el capital como una relación social de producción entre personas y cosas; objetivada en medios de producción, fuerza de trabajo y dinero (Astarita, 2008).

Se trata, por ende, de la relación social e histórica que media entre el capitalista propietario y el trabajador desposeído, tanto más por cuanto el perpetuum mobile de la historia es la lucha de clases. Hic et nunc, la condición necesaria y suficiente para la existencia del orden de sociedad capitalista es que entre el capital y el trabajo medie una relación de dominación cuya expresión genuina sea la explotación de la fuerza de trabajo.

\section{CONCLUSIONES}

En el decurso del estudio de la ley de la explotación descubierta por Marx, se desprende que la contradicción entre el valor de uso y el valor de cambio de la fuerza de trabajo se entrelaza a la teoría de la distribución del ingreso. Más concretamente, el problema de la distribución del ingreso se adscribe al problema de la producción, tanto más cuanto que el salario no podrá jamás crecer hasta alcanzar o sobrepasar el nivel que ponga en peligro la creación de plusvalor; id est, la valorización del capital.

En relación con lo anteriormente expuesto, Mattick (1980) subraya que el monopolio que ejercen los capitalistas 
sobre las condiciones de producción les permite adueñarse de la distribución del producto global entre capital y trabajo.

Así, una vez que los capitalistas se apropian de parte del producto en forma de plusvalor es conditio sine qua non que procuren a los trabajadores el suficiente ingreso que permita la reproducción no solo de la fuerza de trabajo sino también de las condiciones de producción. Dicha relación de clases, escribe Mattick (1980), reviste la envoltura de una relación de valor, por cuanto se encuentra doblegada a los designios de la propiedad privada y el mercado capitalistas.

Ahora bien, es importante remarcar que la teoría del salario de Marx no converge hacia una ley de bronce de los salarios como ocurre en Malthus $(1798,1820)$ y Lasalle $(1863)$. Ha de diferenciarse en este sentido el valor de la fuerza de trabajo del salario propiamente dicho. En tanto que el salario constituye el precio de la fuerza de trabajo, este solamente dependerá de la oferta y la demanda en el mercado laboral.

Empero, al igual que los precios de las demás mercancías, el precio de la fuerza de trabajo posee un centro de gravedad sobre el que habrá de orbitar, a saber: el valor de la cesta de bienes de consumo; id est, el valor de la fuer$z a$ de trabajo. En consecuencia, el límite inferior de los salarios es el valor de los medios de vida necesarios para la reproducción de la fuerza de trabajo, mientras que el límite superior lo constituye la tasa de ganancia máxima que podrán obtener los capitalistas. Esto, en suma, constituye la ley salarial fundamental en la teoría de Marx.

No en vano, es de hacer notar que en el modo capitalista de producción se dan las condiciones generales del aumento de los salarios reales (Rosdolsky, 1968), a saber: la extensión de la duración de la jornada laboral, el aumento de la intensidad normal del trabajo y el desarrollo de la fuerza productiva del trabajo.

Mas, por otra parte, el salario relati$v o$, en cuanto expresión de la participación del trabajo en la creación en el nuevo valor creado por él (Marx, 1849), refleja una tendencia declinante merced al cambio técnico, la elevación del grado de mecanización del proceso productivo, las mejoras de productividad o el avance en el transporte de mercancías.

Dicho de otra forma, tendencialmente la participación de la ganancia del capital en el producto global se ensancha, aun cuando el salario real por trabajador haya crecido. No debe asombrar en modo alguno que Marx $(1956,1959)$ prestase especial atención a la categoría de salario relativo, tanto más cuanto que en realidad constituye otra forma de expresar la teoría de la explotación de la fuerza de trabajo. 


\section{REFERENCIAS}

Amin, S. (1973). La acumulación a escala mundial. Crítica de la teoría del subdesarrollo. Ciudad de México: Siglo XXI.

Astarita, R. (2008). Keynes, poskeynesianos y keynesianos neoclásicos. Buenos Aires: Universidad Nacional de Quilmes.

Baran, P. \& Sweezy, P. (1966). El capital monopolista: ensayo sobre el orden económico y social de Estados Unidos. México: FCE.

Böhm-Bawerk, E. V. (1876). Valor, capital, interés. Madrid: Unión Editorial.

Böhm-Bawerk, E. V. (1891). Teoría positiva del capital. Madrid: Aosta.

Böhm-Bawerk, E. V. (1896). La conclusión del sistema de Marx. En R. Hilferding, E. V. Böhm-Bawerk \& L. V. Bortkiewicz (eds.) Economía burguesa y economía socialista (pp. 29-127). Buenos Aires: Cuadernos de Pasado y Presente.

Boundi, F. (2014). Relaciones de producción y conflicto capital-trabajo en la economía política. Barataria, Revista Castellano-Manchega de Ciencias Sociales, (18), 81-96. https://doi.org/10.20932/barataria.v0i18.45

Emmanuel, A. (1970). El problema del intercambio desigual (respuesta a Palloix). En A. Emmanuel, C. Bettelheim, S. Amin \& C. Palloix (eds.) Imperialismo y comercio internacional. El intercambio desigual (pp. 185-220). Madrid: Siglo XXI.

Grossmann, H. (1929). La ley de la acumulación y del derrumbe del sistema capitalista. México: Siglo XXI.

Gunder, A. (1966). Latin America: Underdevelopment or Revolution. New York: Monthly Review Press.

Hirschman, A. O. (1958). La estrategia del desarrollo económico. México: FCE.

Jevons, S. (1870). Political economy. New York: Kessinger Publishing.

Kalecki, M. (1939). Estudios sobre la teoría de los ciclos económicos. Barcelona: Ariel.

Keynes, J. M. (1933). Robert Malthus (1766-1834). El primer economista de Cambridge. En R. Malthus, Primer ensayo sobre la población (pp. 9-40). Madrid: Alianza. 
Apuntes CENES Volumen 36, Número 64

julio - diciembre 2017. Págs. 15-46

Keynes, J. M. (1936). Teoría general del empleo, el interés y el dinero. México: FCE.

Kicillof, A. (2005). Génesis y estructura de la teoría general de Lord Keynes. Buenos Aires: Universidad de Buenos Aires, Facultad de Ciencias Económicas, Departamento de Doctorado.

Kicillof, A. (2009). Salario, precio y ganancia en la teoría general. Desarrollo Económico, 48(190), 471-513.

Lange, O. (1964). La economía en las sociedades modernas. México: Grijalbo.

Lange, O. (1965). Teoría de la reproducción y de la acumulación. Barcelona: Ariel.

Lassalle, F. (1863). Aiberterprogramm. Zürich: Verlag von Meyer \& Zeller.

Leontief, W. (1951). The Structure of the American Economy, 1919-1939. Harvard: Harvard University Press.

Malthus, R. (1798). Primer ensayo sobre la población. Barcelona: Alianza.

Malthus, R. (1820). Principles of Political Economy Considered with a View of their Practical Application. London: John Murray.

Marshall, A. (1890). Principios de economía. Madrid: Síntesis.

Marx, K. (1849). Trabajo asalariado y capital. Madrid: Ricardo Aguilera.

Marx, K. (1867). El Capital: crítica de la economía política, libro I. México: FCE.

Marx, K. (1882). Glosas marginales al tratado de economía política de Adolfo Wagner. En K. Marx, El Capital: crítica de la economía política, libro I (pp. 713723). México: FCE.

Marx, K. (1885). El Capital: crítica de la economía política, libro II. México: FCE.

Marx, K. (1894). El Capital: crítica de la economía política, libro III. México: FCE.

Marx, K. (1939). Elementos fundamentales para la crítica de la economía política, borrador 1857-1858, Grundrisse, libro I. Madrid: Siglo XXI.

Marx, K. (1956). Teorías sobre la plusvalía, libro I. México: FCE.

Marx, K. (1959). Teorías sobre la plusvalía, libro II. México: FCE.

Mattick, P. (1974). Crisis económica y teorías de la crisis. Madrid: Maia. 
Mattick, P. (1980). Crítica de la teoría económica contemporánea. México: Era.

Morishima, M. (1973). La teoría económica de Marx. Una teoría dual del valor y el crecimiento. Barcelona: Tecnos.

Morishima, M. (1974). The Fundamental Marxian Theorem: A Reply to Samuelson. Journal of Economic Literature, 12(1), 71-74.

Morishima, M. \& Seton, F. (1961, Apr.). Aggregation in Leontief Matrices and the Labour Theory of Value. Econometrica: Journal of The Econometric Society, 29(2), 203-220. https://doi.org/10.2307/1909289

Okishio, N. (1963). A mathematical Note on Marxian Theorems. Weltwirtschaftliches Archiv, (91), 287-299.

Quesnay, F. (1758). Tableau économique des physiocrates. París: Calmann-Lévy.

Ricardo, D. (1821). Principios de economía política y tributación. Madrid: Hora H.

Robinson, J. (1942). Ensayo sobre economía marxista. México: Siglo XXI.

Roemer, J. E. (1986). Valor, explotación y crisis. México: FCE.

Rosdolsky, R. (1968). Génesis y estructura de El Capital de Marx. Estudio sobre los Grundrisse. México: Siglo XXI.

Schumpeter, J. A. (1942). Capitalismo, socialismo y democracia. Barcelona: Orbis.

Schumpeter, J. A. (1954). Historia del análisis económico. Barcelona: Ariel.

Senior, N. W. (1836). An Outline of the Science of Political Economy. New York: Augustus M. Kelley.

Shaikh, A. (1991). Valor, acumulación y crisis. Bogotá: Tercer Mundo.

Shaikh, A. (2016). Capitalism. Competition, conflict, crises. New York: Oxford University Press. https://doi.org/10.1093/acprof:oso/9780199390632.001.0001

Smith, A. (1776). Investigación sobre la naturaleza y causa de la riqueza de las naciones. México: FCE.

Sombart, W. (1913). El burgués. Barcelona: Alianza.

Walras, L. (1874). Éléments deéconomie politique pure ou théorie de la richesse sociale. Nueva York: Nabu Press. 